\title{
MODELLING THE POWER SPECTRUM OF DENSITY FLUCTUATIONS: A PHENOMENOLOGICAL APPROACH
}

\author{
Enzo Branchini ${ }^{1}$, Luigi Guzzo ${ }^{2}$ \& Riccardo Valdarnini ${ }^{1}$
}

Accepted by The Astrophysical Journal (Letters) - December 30, 1993

1 SISSA - International School of Advanced Studies, Strada Costiera 11, I-34014 Trieste, Italy.

2 Osservatorio Astronomico di Brera, Sezione di Merate, Via Bianchi 46, I-22055 Merate (CO), Italy. 


\begin{abstract}
We show how, based on considerations on the observed form of the galaxy two-point spatial correlation function $\xi(r)$, a very simplified - yet surprisingly effective - model for the linear density fluctuations power spectrum can be constructed. We first relate the observed large-scale shape of $\xi(r)$ to a powerlaw form for the power spectrum, $P(k) \propto k^{-2.2}$. For a plausible value of the bias parameter $b=1 / \sigma_{8} \simeq 1.8$, one has $(\delta \rho / \rho)_{r m s} \sim 1$ at $r \simeq 3.5 h^{-1} \mathrm{Mpc}$, suggesting that the change of slope observed in $\xi(r)$ around this scale marks the transition between the linear and nonlinear gravitational regimes. Under this working hypothesis, we use a simple analytical form to fit the large-scale correlations constraints together with the COBE CMB anisotropy measurement, thus constructing a simple phenomenological model for the linear power spectrum. Despite its simplicity, the model fits remarkably well directly estimated power spectra from different optical galaxy samples, and when evolved through an $\mathrm{N}$-body simulation it provides a good match to the observed galaxy correlations. One of the most interesting features of the model is the smallscale one-dimensional velocity dispersion produced: $\sigma_{1 d}=450 \mathrm{Km} \mathrm{s}^{-1}$ at 0.5 $h^{-1} \mathrm{Mpc}$ and $\sigma_{1 d}=350 \mathrm{Km} \mathrm{s}^{-1}$ for separations $\geq 2 h^{-1} \mathrm{Mpc}$.
\end{abstract}

\title{
1. INTRODUCTION
}

Cosmological models for the formation of the large-scale structure of the Universe require as a key ingredient a specific form for the power spectrum of density fluctuations $P(k)$ at recombination. This is usually specified in terms of a primordial spectrum together with a transfer function which describes the subsequent evolution of fluctuations depending on the physical scenario. A paradigmatic example is provided by the Cold Dark Matter (CDM) model (e.g. Blumenthal et al. 1984; White et al. 1987).

Conversely, one might ask, independently of any a priori physical model, whether a specific shape for the power spectrum is implied by the available data, i.e. adopt a phenomenological approach (e.g. Kashlinsky 1992; Scaramella 1992; Taylor \& Rowan-Robinson 1992). Indeed, observations have reached a stage in which it is possible to constrain directly the power spectrum on linear scales, where $(\delta \rho / \rho)_{r m s}<1$ and the recombination shape of $P(k)$ should be preserved. On one side galaxy redshift surveys have allowed reliable estimates of the two-point correlation function $\xi(r)$, the Fourier counterpart of $P(k)$, up to separations $\sim 30 h^{-1} \mathrm{Mpc}$ (de Lapparent, Geller \& Huchra 1988; Guzzo et al. 1991, hereafter G91; Loveday et al. 1992), and direct estimates of $P(k)$ itself (Baumgart \& Fry 1991; Peacock \& Nicholson 1991; Vogeley et al. 1992, hereafter V92; Jing \& Valdarnini 1993, hereafter JV93; Fisher et al. 1993, hereafter FDSYH). On much larger scales, the discovery by the COBE-DMR experiment of temperature fluctuations in the microwave background radiation (Smoot et al. 1992) has produced a further fundamental constraint on the very longwavelength amplitude of $P(k)$. Among the outcomes of this relative wealth of results is the fact that the standard flat CDM model (although successful 
on several grounds), cannot consistently reproduce all the observations. The difficulties arise essentially from a too-high ratio of small-scale to large-scale power. Several modifications to the standard CDM scenario have been explored in the attempt to overcome these problems . Among these, open models with non-vanishing cosmological constant (Efstathiou et al. 1990), models with tilted primordial index (Adams et al. 1992, hereafter A92; Cen et al. 1992), or mixed (CDM plus HDM) models (Davis, Summers \& Schlegel 1992 and references therein). Rather than entering the debate on which physical model is closer to the observed behaviour, in this Letter we prefer to keep to the pure phenomenological approach to reconstruct a "minimal" model for the linear $P(k)$. As we shall see, when we close the circle, despite its naivety the model proves to be remarkably effective in accounting for a number of observations.

\section{A PHENOMENOLOGICAL POWER SPECTRUM}

The most natural way to construct a phenomenological model for the linear $P(k)$ would be to start from its most recent direct determinations (as e.g. V92 or JV93), try to 'clean' the observed shape from the expected non-linear effects, and then add further constraints from other observables. Here, however, we choose to start in an apparently less straightforward way, i.e. from the observed two-point correlation function $\xi(r)$. The reason is that we first want to show how the large-scale drop-off of $\xi(r)$ contains important information and, although not a power-law, can be related to a specific, simple shape of $P(k)$ on intermediate scales. We stress that the phenomenological model obtained from this heuristic procedure will be fully justified only a posteriori, when it will possibly satisfy the main observational constraints.

Let us therefore consider the observed spatial correlation function estimated from redshift survey samples. This is different from the true (real space) one, because on small scales large velocity dispersions within clusters ('Fingers of God') depress its amplitude, while on large scales coherent motions amplify it according to linear theory prescriptions (Kaiser 1987). These effects can be properly accounted for by estimating $\xi\left(r_{p}, \pi\right)$, (e.g. Fisher et al. 1993b), i.e. decomposing the pair separation vector along the directions parallel and perpendicular to the line of sight. A practical, approximate correction of the small-scale distortions can however be performed by collapsing the cluster 'fingers' into a region corresponding to their statistically expected spatial size. Using this method, G91 obtained from the Perseus-Pisces (PP herafter) redshift survey, a correlation function reproducing the canonical real-space shape for optical galaxies $\propto r^{-1.8}$ (Davis \& Peebles 1983), shown in fig. 1. Although this function is still in redshift space at large separations, we prefer to use the notation $\xi(r)$ instead of the conventional $\xi(s)$ to underline the correction to real space operated on small scales. In the same figure we plot (open circles) the more recent estimate (in pure redshift space) from the extension of the CfA redshift survey (V92). Note the the small-scale depression of the latter correlation function and the remarkable agreement of the two samples for $r>2 h^{-1} \mathrm{Mpc}$. In particular, both samples are very well described for separations larger than $r_{b} \simeq 3.5 h^{-1} \mathrm{Mpc}$ by the simple law $\xi(r)=\left[(r / 20)^{-0.8}-1\right]$ (dashed line), where $r$ is in $h^{-1} \mathrm{Mpc}$. 
The consistency of the two surveys further demonstrates that the original finding of G91 cannot be ascribed to peculiarities in the Perseus-Pisces region, and that $r_{b} \simeq 3.5 h^{-1} \mathrm{Mpc}$ seems to represent a physically meaningful scale (see also Calzetti, Giavalisco \& Meiksin 1992).

By Fourier transforming the above expression for $\xi(r)$ - assuming negligible correlations for $s>20 h^{-1} \mathrm{Mpc}$ - we can have a first rough indication of the shape of the corresponding power spectrum: $P(k) \propto k^{-2.2}$. Although $\xi(r)$ is not rigorously a power law at large separations, the corresponding $P(k)$ still seems to be. More accurately, if we adopt a simple functional form as used by Peacock (1991) to fit the APM angular function,

$$
P(k)=\frac{A k^{\alpha}}{1+\left(\frac{k}{k_{c}}\right)^{\alpha-n}}
$$

then we can match the observed correlations for $s>r_{b}$ with $\alpha \simeq-2.2$, $k_{c} \simeq 0.08 h \mathrm{Mpc}^{-1}$ and virtually any value of $n$ in the range $[0,1]$ (which has very little effect on $\xi$ for $\lambda<2 \pi / k_{c}$ ). At this point we have essentially two ways to constrain the primordial index $n$. The first is simply to assume an Harrison-Zel'dovich primordial spectrum, i.e. $n=1$. In this case, considering the microwave background $\mathrm{rms}$ temperature fluctuation on $10^{\circ}$ scales measured by $\mathrm{COBE}, \sigma_{T}\left(10^{\circ}\right)=[1.085 \pm 0.183] \times 10^{-5}$ (Smoot et al. 1992$)$, we obtain a normalization corresponding to a bias factor $b=1 / \sigma_{8} \simeq 1.4$, where $\sigma_{8}$ is the mass variance in a top-hat window of $8 h^{-1} \mathrm{Mpc}$ radius. For clarity, note that we are using here the conventional definition of $b=\sigma_{8}(\mathrm{gal}) / \sigma_{8}(\mathrm{mass})$, together with the Davis \& Peebles (1983) result that gives $\sigma_{8}(\mathrm{gal}) \simeq 1$. One may define a bias factor for bright galaxies, $b^{*}$, compatible with the correlation functions of fig. 1, considering that, once the redshift space amplification is corrected using Kaiser $(1987)$ formula, is $\sigma^{*}{ }_{8}(\mathrm{gal}) \simeq 1.3$ and therefore $b^{*} \simeq 1.3 b$.

A completely different route to get a plausible value of the $(n, b)$ pair is to note that if $b=1.8-2$, a range which turns out to be justified in several respects for optical galaxies, then we have $\sigma_{R} \sim 1$ for $R \simeq 3.5 h^{-1} \mathrm{Mpc}=r_{b}$. In other words, in such a case the change of slope in the observed correlation function is located right around the scale where $(\delta \rho / \rho)_{r m s} \sim 1$, i.e. coincides with the expected transition from the linear to the nonlinear clustering regimes. This has the important consequence that larger scales should still be evolving in a quasi-linear fashion and could therefore constrain directly the shape of the initial spectrum. Using this kind of bias value, and considering again the COBE constraint at large wavelengths we obtain $n \simeq 0.75$ if we neglect the gravitational wave contribution to the Sachs-Wolfe effect (Lucchin, Matarrese \& Mollerach 1992; A92). We note that in this case all the important parameters of the spectrum, i.e. $b \simeq 1.9, \alpha \simeq-2.2$ and $n \simeq 0.75$ are globally consistent with those of a correspondingly tilted CDM spectrum (Cen et al. 1992; A92). This is evident in fig. $2 \mathrm{a}$, where we compare our euristic fit with CDM models of varying primordial index $(n=0.7,0.8,0.9,1)$, adopting the Bardeen et al. (1986, BBKS) CDM transfer function. It is implicit in this comparison that we are considering our large-scales fit as representative of the linear power spectrum, in particular 
that any residual redshift space effects are limited to the Kaiser amplification. This is quite a strong assumption and might be questionable in several ways (e.g. FDSYH; Bahcall, Cen \& Gramann 1993). However, let us assume here as a working hypothesis that expression (1), with $\alpha=-2.2, n=0.75-1$, $b=1.4-1.9$, represents a good linear phenomenological description of the true $P(k)$. We shall call it the Phenomenologically Induced Model (PIM). The remainder of the paper will concentrate on comparing it with the observations, and possibly narrowing the constraints on its normalization and primordial index.

\section{LINEAR AND NONLINEAR TESTS}

The first test is obviously to check whether our phenomenological form is a good representation of the 'general' behaviour of clustering, as quantified by power spectra measurements on independent samples. To this end, in fig. $2 \mathrm{~b}$ the PIM, with $n=0.75$ and $n=1$ and normalized to fit the observed largescale $\xi(r)$ of the PP sample, is compared to the direct estimate of $P(k)$ from the CfA survey (V92). This comparison is particularly significant, since both surveys are constructed starting from the same photometric material (the Zwicky catalogue), and both the PP $\xi(r)$ and the CfA $P(k)$ are estimated on volumelimited samples with $M$ brighter than $\sim M^{*}$. For these reasons we prefer to avoid including in the figure measures of $P(k)$ for, e.g., radio galaxies (Peacock \& Nicholson 1991), or IRAS galaxies (FDSYH; JV93), which inevitably require an arbitrary amplitude renormalization to be compared with the optical data (see fig. 1 of V91 for an indirect comparison). The agreement of the PIM with the CfA $P(k)$ is evidently very good. Note that there is no renormalization between the curve and the data points.

What seems to be worrying in fig. $2 \mathrm{~b}$ is that the data points continue to follow the PIM curve - which is supposed to be describing the linear $P(k)$ also for for $k>0.3 h M p c^{-1}$, where nonlinear effects certainly start to become significant. To understand this, we have Fourier-transformed the PP $\xi(r)$ of fig. 1, which is corrected for the main nonlinear distortions produced by the 'Fingers of God'. The result is plotted as a dashed line in in fig. 2b, showing that, as one expects from the small-scale $r^{-1.8}$ shape of the correlation function, $P(k)$ rises to $\sim k^{-1.2}$ as consequence of nonlinear clustering. It is interesting to notice, therefore, that there seems to be a kind of 'conspiracy': nonlinear clustering enhance correlations at small-scales, but when these are viewed in redshift space, the global effect of small-scale velocity dispersions seems to bring the spectrum back to a shape similar to the purely linear one. This is the same effect visible in the correlation function (fig. 1): when the 'finger of God' effect is not corrected $(\mathrm{CfA}), \xi(r)$ is well approximated by a single law $\propto\left[(r / 20)^{-0.8}-1\right]$ from small to large scales.

The other important test is to check how the PIM behaves when nonlinear gravitational evolution sets in. To this end, we have performed an N-body simulation, concentrating our attention on two of the most crucial observational tests: the two-point angular correlation function $w(\theta)$, and the one-dimensional galaxy velocity dispersion $\sigma_{1 d}$. We do not perform here a detailed investigation 
of redshift-space clustering (as e.g. in FDSYH), which is left for a more comprehensive paper involving further simulations with extended dynamic range.

For our specific aim, this first simulation was planned as a compromise between the necessity of a large box size, required by the considerable amount of large-scale power present in the PIM spectrum $\left(L_{b o x}>2 \pi / k_{\text {turn }} \sim 130 h^{-1}\right.$ $\mathrm{Mpc}$ ), and the need of a good spatial resolution for not to underestimate $\sigma_{1 d}$. We therefore used a $\mathrm{P}^{3} \mathrm{M}$ code to integrate the motion of $64^{3}$ particles within a $180 h^{-1} \mathrm{Mpc}$ sided box, with $160^{3}$ grid points and a resolution of $0.3 h^{-1} \mathrm{Mpc}$. We used $\Omega_{o}=1$ and $H_{o}=50 \mathrm{~km} \mathrm{~s}^{-1} \mathrm{Mpc}^{-1}$. Initial conditions were described by a Gaussian random field, and the 'galaxies' were selected using the BBKS prescription, as in White et al. (1987).

A further important reason for performing an N-body test is to possibly put some more stringent constraints on $n$ and $b$. To this end, we adopted the following procedure. We did not consider the COBE normalization to fix the present epoch, but leave clustering evolve until the small-scale shape of $\xi(r)$ for 'bright galaxy peaks' (defined with a threshold corresponding to $\nu_{\text {sim }}=1.3 b \simeq$ $2.3)$ in the simulation matched the observed one. At this point $b=1 / \sigma_{8} \simeq$ 1.8 , and the corresponding $n$ required to match COBE with the PIM form is $n \simeq 0.85$. Fig. 1 shows the two-point correlation function for the simulated galaxies (solid line), calculated in real space as in White et al. 1987. In order to be compared with the PP estimate, $\xi(r)$ has been multiplied by the proper linear amplification factor (Kaiser 1987), on the scales where $(\delta \rho / \rho)_{r m s}<1$. The match with the observations is remarkable in both shape and amplitude over a wide range of scales, a result which seems to support nicely the idea that the change of slope at $r_{b} \simeq 3.5 h^{-1} \mathrm{Mpc}$ tags the transition scale to full nonlinearity.

A crucial test for the model is provided by the angular correlation function $w(\theta)$, which is by definition independent from any redshift space effect. We estimated $w(\theta)$ by projecting $\xi(r)$ of the simulation through the Limber equation, adopting a selection function and normalization corresponding to the Lick depth (see Peacock 1991). Since at large separations $\left(r>15 h^{-1} \mathrm{Mpc}\right)$ the single simulation performed shows slightly less power than the linear model, on these scales we used $\xi(r)$ from the latter one, smoothly joined to the numerical result at smaller scales. The final result is shown in fig. 3, compared with the APM (Maddox et al. 1990), and EDSGC (Nichol \& Collins 1993) data. The PIM spectral shape does still seem to lack some power at very large angular separations. This might either suggest that the spatial estimates of $\xi(r)$ and $P(k)$, on which the PIM is based, still suffer from the limited sample size, or be related to possible sistematics in $w(\theta)$ at these large angles.

The other main aim of the simulation was to check the small-scale velocity dispersion produced by the PIM. We computed $\sigma_{1 d}(r)$ for the dark particles as $\sigma_{1 d}(r)=1 / \sqrt{3}\left\langle\left|\mathbf{v}_{1}-\mathbf{v}_{2}\right|\right\rangle^{1 / 2}$, where $\mathbf{v}_{1}$ and $\mathbf{v}_{2}$ are the peculiar velocities of the two particles with separation $r$. We obtained $\sigma_{1 d}\left(0.5 h^{-1} \mathrm{Mpc}\right) \simeq 450 \mathrm{Km} \mathrm{s}^{-1}$, rapidly decreasing to $\sim 350$ for $r \geq 2 h^{-1} \mathrm{Mpc}$. This compares interestingly with the observed $\sigma_{1 d} \simeq 320 \mathrm{Km} \mathrm{s}^{-1}$ on $1 h^{-1}$ Mpc scales (Fisher et al. 1993b).

In summary, we have shown that an extremely simplified toy model with a 
few specific features, in particular a steep slope $\sim k^{-2.2}$, a turnover around $k \sim$ $0.05 h \mathrm{Mpc}^{-1}$ and a biasing $b \sim 1.8$, is capable of reproducing remarkably well some of the main properties of galaxy clustering. This supports the soundness of the basic assumptions made for its construction, in particular the idea that fluctuations on scales larger than $\sim 5 h^{-1} \mathrm{Mpc}$ are still weakly affected by nonlinear evolution. Clearly, more extended tests are required, in particular direct comparison of real and redshift space properties from several simulated catalogues. Also, we have not touched here the issue of which physical scenarios might be able to produce a spectrum at recombination with this form. However, it seems that this first phenomenological investigation has at least underlined a few features which seem to be unavoidable for any physical model aimed at explaining the observed large-scale structure. During the revision of this paper, we became aware of a work by Baugh \& Efstathiou (1993), who accurately determine $P(k)$ in true real space by de-projecting the APM $w(\theta)$. Their result fully agrees with our claim that the power spectrum has a steep range $\sim k^{-2}$ for $0.08<k<0.3 h \mathrm{Mpc}^{-1}$ which is not the result of redshift-space effects, and raises to $k^{-1.2}$ for $k>0.3 h M p c^{-1}$ due to the onset of nonlinear clustering (cf. our fig. 2b with, e.g., their fig. 12).

\section{Acknowledgments}

We thank J.R. Bond, S. Bonometto, R. Carlberg, A. Klypin, L. Kofman, F. Lucchin, J. Peacock, A. Provenzale and M. Scodeggio for fruitful discussions, P. Haines for reading the manuscript, S. Maddox, R. Nichol and M. Vogeley for providing us with a computer version of their results, CINECA and ICTP for computing time allocation, and GNA/CNR for financial support. EB is expecially grateful to P. Catelan and S. Matarrese for their precious suggestions, to SISSA for financial support and to the Department of Astronomy of the University of Toronto for hospitality during the final phases of this work. The referee, M. Strauss, is gratefully acknowledged for the many important suggestions which greatly improved the presentation of this work.

\section{References}

Adams, F. C., Bond, J. R., Freese, K., Frieman, J. A., \& Olinto, A. V., 1993, Phys.Rev., D47, 426 (A92).

Bahcall, N.A., Cen, R., \& Gramann, M., 1993, ApJ, 408, L77

Bardeen, J.M., Bond, J.R., Kaiser, N., \& Szalay, A.S., 1986, ApJ, 304, 15.

Baugh, C.M., \& Efstathiou, G.P., 1993, MNRAS, 265, 145.

Baumgart, D.J., \& Fry, J.N., 1991, ApJ, 375, 25.

Blumenthal, G.R., Faber, S.M., Primack, J.R., \& Rees, M.J., 1984, Nature , $311,517$.

Calzetti, D., Giavalisco, M. \& Meiksin, A., 1992, ApJ, 398, 429.

Cen, R., Gnedin, N. Y., Kofman, L. A. \& Ostriker, J. P., 1992, ApJ, 399, L11.

Cen, R. \& Ostriker, J. P., 1993, preprint.

Davis, M. \& Peebles, P. J. E., 1983, ApJ, 267, 456.

Davis, M., Summers, F. S. \& Schlegel, D. 1992, Nature, 359, 393.

de Lapparent, V., Geller, M. J., \& Huchra J. P., 1988, ApJ, 324, 44.

Efstathiou, G., Sutherland, W. S. \& Maddox, S. J. 1990, Nature, 348, 705. 
Fisher, K. B., Davis, M., Strauss, M. A., Yahil, A. \& Huchra, J. P., 1993, ApJ, 402, 42 (FDSYH).

Fisher, K. B., Davis, M., Strauss, M. A., Yahil, A. \& Huchra, J. P., 1993b, MNRAS, in press.

Guzzo, L., Iovino, A., Chincarini, G., Giovanelli, R. \& Haynes, M. P., 1991, ApJ, 382, L5. (G91).

Hudson, M.J., 1993, MNRAS, submitted

Jing, J. P. \& Valdarnini, R., 1993, ApJ, 406, 6 (JV93).

Kaiser, N., 1987, MNRAS, 227, 1.

Kashlinsky, A., 1992, ApJ, 387, L1

Loveday, J., Peterson, B. A., Efstathiou, G. \& Maddox, S. J., 1992, ApJ, 390,388 .

Lucchin, F., Matarrese, S., \& Mollerach, S., 1992, ApJ, 401, L49.

Maddox, S. J., Efstathiou, G., Sutherland, W. J. \& Loveday, J., 1990, MNRAS, $242,43 p$.

Nichol, R.C., \& Collins, C.A., 1992, MNRAS, in press.

Peacock, J. A., 1991, MNRAS, 253, 1P.

Peacock, J. A. \& Nicholson, D., 1991, MNRAS, 253, 307.

Scaramella, R., 1992, ApJ, 390, L57.

Smoot, G. F. et al. , 1992, ApJ, 371, L1.

Taylor, A. N. \& Rowan-Robinson, M., 1992, Nature , 359, 396.

Vogeley, M. S., Park, C., Geller, M. J. \& Huchra, J. P., 1992, ApJ, 391, L5 (V92).

White, S.D.M, Frenk, C.S., Davis, M., \& Efstathiou, G., 1987, ApJ, 313, 505. 


\section{Figure captions}

\section{Figure 1.}

Two-point correlation functions from the PP (G91) and CfA (V92) redshift surveys. The CfA estimate is in pure redshift space, while the PP sample has been statistically corrected for the small-scale 'Finger of God' effect. Error bars are $1-\sigma$ bootstrap errors. The dashed line shows the curve $\xi(r)=(r / 20)^{-0.8}-1$, while the solid line is the result of the $\mathrm{P}^{3} \mathrm{M}$ N-body integration of the PIM.

\section{Figure 2.}

a) The power spectrum of the phenomenologically induced model (PIM), with primordial index fixed to $n=0.75$, normalized to COBE and compared to flat CDM models with varying primordial index $(n=0.7,0.8,0.9,1)$.

b) The PIM power spectrum normalized to match the PP galaxy correlations of Fig. 1 (solid line) for the two cases $n=0.75$ and $n=1$, compared to the direct estimate of $P(k)$ from the CfA survey by V92 (circles). The dashed line shows the result of Fourier transforming the observed PP small-scale $\xi(r)$.

\section{Figure 3.}

Angular correlation function $w(\theta)$ calculated from the $\mathrm{N}$-body integration of the PIM (solid line), compared with the APM (dots) and EDSGC (circles) data. The EDSGC $1 \sigma$ error bars show the large uncertainty existing in $w(\theta)$ for $\theta>10^{\circ}$. 


\section{Postal and e-Mail Addresses}

Enzo Branchini

SISSA, Strada Costiera 11,

I-34014 TRIESTE - ITALY

e-mail: enzo@tsmi19.sissa.it

Luigi Guzzo

Osservatorio Astronomico di Brera, Sezione di Merate

Via Bianchi 46, I-22055 MERATE (CO) - ITALY

e-mail: guzzo@astmim.mi.astro.it

Riccardo Valdarnini

SISSA, Strada Costiera 11,

I-34014 TRIESTE - ITALY

e-mail: valda@tsmi19.sissa.it 